\title{
A new duty-cycling approach for 3D needle steering allowing the use of the classical visual servoing framework for targeting tasks
}

\author{
Alexandre Krupa
}

\begin{abstract}
We present a method for three dimensional steering of a beveled-tip flexible needle that can be used in medical robotics for percutaneous assistance procedures. The proposed solution is based on the design of a new duty-cycling control strategy that makes possible to control three degrees of freedom (DOFs) of the needle tip. These DOFs correspond to two rotations that are performed around the needle tip lateral axes and one translation along the needle shape axis. This approach has two advantages compared to existing methods. First it does not rely on a trajectory planning and opens therefore numerous closed-loop control scheme possibilities as for example the implementation of the visual servoing framework based on the task function approach. Second, it does not constraint the needle to follow a succession of planar arcs but allows non planar 3D trajectories. Experimental results of a targeting task performed by visual servoing demonstrate the feasibility of this new concept and its robustness to needle kinematic model errors.
\end{abstract}

\section{INTRODUCTION}

Physicians perform needle insertion into human tissues for various medical interventions such as biopsy or injecting therapeutic drugs into local tumours. This kind of operations requires a lot of skills from the clinician in order to reach the target successfully. In fact, positioning errors with respect to the target can imply injuries of tissues and compromise the effectiveness of diagnosis or therapy, or bias the results of studies that require accurate needle placement. That is the reason why robotic assistance for needle steering is a fascinating subject of ongoing research as reported in [1]. The needles can be either rigid or very flexible depending on the medical applications considered. In practice, all needles tend to bend when they are inserted due to the forces that the tissues exert on them. This bending effect is strongly increased for flexible needle and becomes an advantage for targeting anatomical elements that were not possible to reach with a standard rigid needle. In fact, bending the needle can be very useful in order to avoid obstacles such as sensitive organs or bones. The material of this type of needle is flexible and its tip is beveled so that the tissues exert an asymmetrical force on the needle tip causing it to bend naturally. In opposite, the tip of a standard rigid needle is symmetric, and the material used is generally stiff. A flexible needle with asymmetric beveled tip, when inserted straight forward follows a circular arc trajectory [2], [3]. The trajectory taken depends on the geometry of the beveled tip and on the relative stiffness between the needle and the tissue. In order to steer the needle, it is needed to use a kinematic model.

Alexandre Krupa is with Inria Rennes - Bretagne Atlantique and IRISA, France, alexandre.krupa at inria.fr
Generally the model considered in most of research studies related to needle steering is the one of a bicycle or a unicycle [2]. An alternative approach was also proposed in [4] where the needle is modeled as a linear beam supported by virtual springs. In all existing approaches a path planning initial step is required to predict the needle trajectory before steering it. The planner must take into account the nonholonomic constraints of the needle in order to work. Different solutions were proposed to reach this goal. Most approaches use inverse kinematics to plan paths in $2 \mathrm{D}$ or $3 \mathrm{D}$ [5]. In [6] a finite element mesh is used to predict soft tissue deformations and the needle path planning is done thanks to numerical optimization. Probabilistic methods were also tested, like the Rapidly-Exploring-Random Tree (RRT) algorithm [7], or the Stochastic roadmap [8]. This last one gives very good results for preoperative path planning, however it requires a lot of computing and therefore cannot be used to adapt the path in real-time intraoperatively.

Adjusting the path intraoperatively in a control-loop has been done in [9] for needle steering in a 2D plane thanks to the use of an extented Kalman filter that estimates the current pose of the needle from image feedback. The path planning consists then in computing a planar arc-trajectory of the needle that links the current tip position to the target thanks to the use of the inverse kinematic model of the needle. This path is then used to determine the input of a duty-cycling control technique [10] that is applied to steer the needle along the desired arc-trajectory. Other approaches based on the RRT algorithm were proposed to take into account obstacles during the intraoperative path planning. For example, a RRT algorithm with backchaining was presented in [11] to steer the needle in 3D with obstacle avoidance. More recently, a combination of the RRT algorithm and duty-cycling control technique was proposed in [12] for needle steering in a 2D plane. In this last work the 2D path planning is updated from the current needle tip position. This later is automatically tracked in an image provided by a camera observing the needle evolving in a translucent phantom.

In this paper, we propose a new approach to steer a needle in 3D that has several advantages. First, it does not require a trajectory planning that can be time consuming. Second, it does not constraint the needle to follow a succession of planar arcs but allows non planar 3D trajectories. Third, with the proposed method the direct control of the lateral angular velocities $\omega_{x}, \omega_{y}$ and the insertion velocity $v_{z}$ of the needle extremity is possible. This last point is crucial since, in opposite to path planning approaches, it opens numerous control scheme possibilities as for example visual control 
using the classical framework of visual servoing [13] that is based on the task function approach [14]. This work is a preliminary study toward the development of a needle steering robotic system using 3D ultrasound feedback. In our previous work [15], in collaboration with the authors of [12], we already addressed the compensation of the patient physiological motion by stabilizing the ultrasound image thanks to an ultrasound visual servoing technique [16]. We also recently proposed in [17] an efficient image processing method to automatically detect and track in real-time a flexible needle in a sequence of ultrasound volumes.

The paper is organized as follows. Section II presents the kinematic model of the flexible needle considered in our study and section III recalls the principle of the classic dutycycling control technique allowing to bend the needle with a desired curvature in a plane. We present in Section IV our new 3D duty cycling strategy that allows to control the two lateral angular velocities and the insertion velocity of the needle. Section V presents the control scheme we propose for automatic soft tissue targeting using visual servoing. Finally, experimental results on the visual targeting task are reported and discussed in section VI.

\section{NeEdLE KINEMATIC MODEL}

\section{A. Needle natural bending}

The bevel-tip needle can be driven by applying two velocity inputs to the needle base. These velocities are the insertion speed and the angular spin velocity. The rotational velocity causes the needle to turn about its shaft, reorienting the bevel of the tip. If we neglect the torsional compliance of the needle, the tip rotates at the same speed as the needle base. As previously mentioned, when only a translation motion is applied to the needle, the soft tissue exerts asymmetric forces on the beveled-tip that deflect the needle trajectory. As a consequence the needle follows an arc in a 2D plane. Moreover, if the needle is appropriately selected in terms of material compared to the tissue mechanical properties (needle less stiff than the tissue), the needle shaft trajectory will be the same as the needle tip. Thus, tracking the position of the needle can be performed by tracking only the needle tip.

The needle can be modeled as a nonholonomic vehicle owning 2 degrees of freedom DOF (forward translation and rotation around its shaft). In fact, as experimentally demonstrated in [2], inserting the needle straight forward in soft tissue is equivalent to ride an unicycle along a planar circular arc of constant radius $\mathrm{r}=\frac{1}{K}$ as illustrated in Figure 1. $K$ is the natural curvature of the needle which depends both on needle and tissue mechanical properties. Applying a rotation around the needle shaft allows to reorientate the plane where the needle will further evolve.

\section{B. Nonholonomic constraints}

Let $\mathcal{F}_{n}$ and $\mathcal{F}_{w}$ be respectively a Cartesian frame attached to the needle tip and the world frame as shown in Figure 1. We describe the $3 \mathrm{D}$ pose $\mathbf{p}$ (with $\mathbf{p} \in \mathrm{SE}(3)$ ) of the needle tip

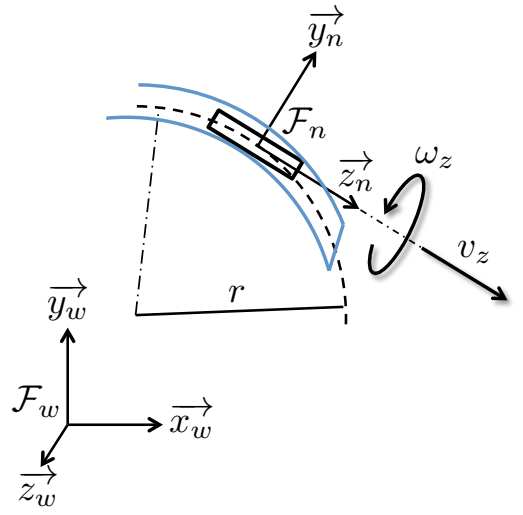

Fig. 1. Needle beveled-tip modeled by an unicycle following an arc of radius $r$.

with respect to $\mathcal{F}_{w}$ by the homogeneous $4 \times 4$ transformation matrix :

$$
{ }^{w} \mathbf{M}_{n}=\left[\begin{array}{cc}
{ }^{w} \mathbf{R}_{n} & { }^{w} \mathbf{T}_{n} \\
\mathbf{0}_{[1 \times 3]} & 1
\end{array}\right]
$$

where ${ }^{w} \mathbf{R}_{n}$ is a $3 \times 3$ rotation matrix representing the orientation of $\mathcal{F}_{n}$ with respect to $\mathcal{F}_{w}$ and ${ }^{w} \mathbf{T}_{n}$ is the $3 \times 1$ translation vector defining the origin of $\mathcal{F}_{n}$ in $\mathcal{F}_{w}$. We also denote the instantaneous velocity of the needle frame $\mathcal{F}_{n}$ with respect to the world $\mathcal{F}_{w}$ by the velocity screw vector: ${ }^{(n)} \mathbf{v}_{n}=\left({ }^{(n)} v_{x},{ }^{(n)} v_{y},{ }^{(n)} v_{z},{ }^{(n)} \omega_{x},{ }^{(n)} \omega_{y},{ }^{(n)} \omega_{z}\right)$. Note that the upper script ${ }^{(n)}$ indicates that this velocity is expressed in the frame $\mathcal{F}_{n}$. Here ${ }^{(n)} v_{x},{ }^{(n)} v_{y},{ }^{(n)} v_{z}$ are the translation velocities along each axis of $\mathcal{F}_{n}$, and ${ }^{(n)} \omega_{x},{ }^{(n)} \omega_{y},{ }^{(n)} \omega_{z}$ are the angular velocities around each axis.

When applying a forward insertion motion to the needle, this later can only move in the plane y-z of $\mathcal{F}_{n}$. Thus, the velocity components ${ }^{(n)} v_{x},{ }^{(n)} v_{y},{ }^{(n)} \omega_{y}$ are constraint to be null and only the insertion velocity ${ }^{(n)} v_{z}$, and angular spin velocity ${ }^{(n)} \omega_{z}$ can be applied by the user to steer the needle. The bending effect introduces however a rotation velocity around the $\mathrm{x}$ axis of $\mathcal{F}_{n}$ that depends on the insertion velocity and natural curvature of the needle such as $\omega_{x}=K^{(n)} v_{z}$. The screw velocity vector is therefore linked to the needle input velocities by the following kinematic relation that expresses the nonholonomic contraints:

$$
{ }^{(n)} \mathbf{v}_{n}=\left[\begin{array}{cccccc}
0 & 0 & 1 & K & 0 & 0 \\
0 & 0 & 0 & 0 & 0 & 1
\end{array}\right]^{T}\left(\begin{array}{l}
{ }^{(n)} v_{z} \\
{ }^{(n)} \omega_{z}
\end{array}\right)
$$

\section{DUTY-CYCLING FOR STEERING INSIDE A PLANE}

\section{A. Principle}

The duty cycling method [10] was introduced to allow the proportional control of the curvature of a bevel-tip needle during insertion. It works by alternating between time period $T_{\text {trans }}$ of pure insertion where the needle will follow an arc of maximum curvature $K$, with time period $T_{\text {rot }}$ of both insertion and rotation, where the needle will follow a helical curve. This later becomes a straight line if the rotation velocity is highly superior than the insertion velocity. Those two phases compose a cycle of period $T=T_{\text {rot }}+T_{\text {trans }}$. 
The ratio of the rotation period $T_{\text {rot }}$ to the cycle period $T$ is defined by:

$$
D C=\frac{T_{r o t}}{T}
$$

With this technique the effective curvature $K_{\text {eff }}$ that will take the needle is given by the following formula:

$$
K_{e f f}=K(1-D C)
$$

Therefore, it is possible to set the curvature of the needle between 0 and the natural curvature $K$. In previous works [10], [12] this method has been implemented by moving during each cycle the needle on a constant fixed insertion distance $\Delta z$, and by applying a constant rotational velocity $\omega_{z}=\omega_{c}$. As a result, the cycle can be defined by:

$$
T_{\text {rot }}=\frac{2 \pi}{\omega_{c}}, T_{\text {trans }}=\frac{T_{\text {rot }}(1-D C)}{D C}, v_{z}=\frac{\Delta z}{T}
$$

\section{B. Discussion on the limitations}

With this technique the needle does a complete rotation ( $2 \pi \mathrm{rad}$ ) during each cycle of period $T$ which allows it to stay in the working plane. However, using this technique restricts the needle to evolve only in a plane. To overcome this limitation some colleagues proposed in [18] to steer the needle along a 3D trajectory composed from a succession of 2D planar arcs. The principle is to use a path planner based on a RRT algorithm that pre-computes from the current needle position and the target position a succession of planar arcs with their relative orientation. The needle is then steered by a stop and turn technique which consists at the starting point of each new arc to first stop the needle and rotate it about its z-axis. This allows to re-orientate the needle beveled tip in the new planned working plane before applying the duty cycling technique. However, this approach is computationally expensive since it requires to perform an initial path planning and update it during the process in order to compensate the model errors . Moreover, the trajectory is constrained to follow a succession of planar arcs that may result to important bends at the arc junction points if the planning is not frequently updated.

\section{3D DUTY-CYCLING NEW STRATEGY}

\section{A. Method for controlling the needle velocities $\omega_{x}, \omega_{y}, v_{z}$}

The principle relies on a modification of the duty-cycling technique in order to allow continuous control of the velocity components $\omega_{x}, \omega_{y}$ and $v_{z}$ of the needle. Let us consider a new cartesian frame $\mathcal{F}_{\tilde{n}}$ attached to the needle that corresponds to the current needle frame rotated with an angle $\theta$ around the $z$ axis of $\mathcal{F}_{n}$. By expressing the rotational velocity of $\mathcal{F}_{\tilde{n}}$ in $\mathcal{F}_{n}$ and using the relation (2) with the effective curvature $K_{\text {eff }}$ we obtain the following relation that links the angular velocity of $\mathcal{F}_{n}$ to the needle input velocities ${ }^{(\tilde{n})} v_{z}$ and ${ }^{(\tilde{n})} \omega_{z}$ expressed in $\mathcal{F}_{\tilde{n}}$ :

$$
\left(\begin{array}{c}
{ }^{(n)} \omega_{x} \\
{ }^{(n)} \omega_{y} \\
{ }^{(n)} \omega_{z}
\end{array}\right)=\left[\begin{array}{ccc}
\cos (\theta) & -\sin (\theta) & 0 \\
\sin (\theta) & \cos (\theta) & 0 \\
0 & 0 & 1
\end{array}\right]\left(\begin{array}{c}
K_{e f f}{ }^{(\tilde{n})} v_{z} \\
0 \\
{ }^{(\tilde{n})} \omega_{z}
\end{array}\right)
$$

As $\mathcal{F}_{\tilde{n}}$ and $\mathcal{F}_{n}$ have the same origin and their z-axis are aligned, we have ${ }^{(n)} v_{z}={ }^{(\tilde{n})} v_{z}$ that we can substitute in (6) in order to formulate the angular velocity of $\mathcal{F}_{n}$ in function of the needle translational velocity and effective curvature:

$$
\begin{aligned}
{ }^{(n)} \omega_{x} & =\cos (\theta) K_{e f f}{ }^{(n)} v_{z} \\
{ }^{(n)} \omega_{y} & =\sin (\theta) K_{\text {eff }}{ }^{(n)} v_{z}
\end{aligned}
$$

One can notice that it is therefore possible to control the ratio between the velocities ${ }^{(n)} \omega_{x}$ and ${ }^{(n)} \omega_{y}$ at each cycle period $T$ by applying a given value of angle $\theta$ around its $\mathrm{z}$-axis before inserting the needle. Section IV-B will describe how this angle accumulation is performed in practice during the duty-cycling. Now, we detail the computation of the desired curvature value $K_{\text {eff }}^{*}$, the desired angle $\theta^{*}$ that represent with ${ }^{(n)} v_{z}^{*}$ the control inputs of the duty cycling low-level controller. We denote by $\left({ }^{(n)} v_{z}^{*},{ }^{(n)} \omega_{x}^{*},{ }^{(n)} \omega_{y}^{*}\right)$ the desired control velocity values that we want to apply to the needle frame. Dividing the second line by the first line of (7) leads directly to :

$$
\theta^{*}=\operatorname{atan}\left({ }^{(n)} \omega_{y}^{*} /{ }^{(n)} \omega_{x}^{*}\right)
$$

and from simple calculus on (7) we obtain:

$$
K_{e f f}^{*}=\left(\sqrt{(n) \omega_{x}^{* 2}+{ }^{(n)} \omega_{y}^{* 2}}\right) /{ }^{(n)} v_{z}^{*}
$$

Note that if ${ }^{(n)} v_{z}^{*}$ is null we set $K_{\text {eff }}^{*}=0$ since the needle is not moving in this case.

\section{B. Duty cycling low-level controller with angle accumulation}

The duty-cycling classic technique introduced in section III-A is not so easy to implement in practice since it is not possible to accurately apply a constant rotation velocity ${ }^{(n)} \omega_{z}=\omega_{c}$ during a given time $T_{\text {rot }}$ and stop immediately the rotation when the spin angle reaches $2 \pi$ rad before beginning the pure translation phase $T_{\text {trans }}$. In fact, performing like this would require a robotic system with a rotating actuator that provides an infinite acceleration to start and stop the needle rotation without generating transient phases. Therefore to cope with this important issue, we propose in our new 3D duty-cycling approach to implement a positionbased controller that regulates the needle spin angle in order to accurately follow a desired angular trajectory. It is based on a motion profile generator that computes in real-time a trapezoidal acceleration profile that is applied to the PID controler of the actuator rotating the needle. The advantage of this position-based controller is to avoid any angular drift on the rotation around the z-axis. In fact, this is crucial for accurately controlling the needle spin angle during the duty cycling process. With this approach, the desired rotation velocity ${ }^{(n)} \omega_{z}^{*}$ is not constant during the acceleration and deceleration phases of the rotating actuator but an accurate final angle positioning is achieved.

Here we detail the successive steps that are performed during one period of our modified duty-cycling approach that allows the application of the velocity control inputs $\left({ }^{(n)} v_{z}^{*},{ }^{(n)} \omega_{x}^{*},{ }^{(n)} \omega_{y}^{*}\right)$ to the needle frame $\mathcal{F}_{n}$.

1) measure the current absolute needle spin angle: $\theta_{a}$ 
2) compute the desired absolute spin angle to achieve by accumulating $2 \pi \mathrm{rad}$ and the control angle input $\theta^{*}$ given by eq. (8) : $\theta_{a}^{*}=\theta_{a}+2 \pi+\theta^{*}$

3) compute the desired period $T_{\text {rot }}^{*}$ and desired spin angular velocity profile ${ }^{(n)} \omega_{z}^{*}$ needed to achieve $\theta_{a}^{*}$ based on the trapezoidal acceleration profile with a nominal needle spin velocity of $\omega_{c}$

4) compute the desired insertion distance $\Delta z^{*}={ }^{(n)} v_{z}^{*} T_{\text {rot }}^{*}$

5) perform the rotation and insertion thanks to the positionbased robot controller to reach the desired angle $\theta_{a}^{*}$ and translation $\Delta_{z}^{*}$

6) measure the real period $T_{\text {rot }}$ that was taken by the system

7) compute the desired cycle ratio $D C^{*}=1-K_{\text {eff }}^{*} / K$ with $K_{\text {eff }}^{*}$ given by (9), the desired pure translation period $T_{\text {trans }}^{*}=T_{\text {rot }}\left(1-D C^{*}\right) / D C^{*}$ and the desired insertion distance $\Delta z^{*}={ }^{(n)} v_{z}^{*} T_{\text {trans }}^{*}$

8) perform the insertion thanks to the position-based robot controller to reach the desired translation $\Delta_{z}^{*}$

9) go back to step 1) to start a new cycle.

In practice, we also add a saturation mechanism on the desired curvature value $K_{\text {eff }}^{*}$ in order to guarantee that the pure translation phase $T_{\text {trans }}$ will not take infinite time. In fact this could happen when the desired curvature is not reachable due to the limitation of needle natural curvature $K$. We propose therefore to saturate $K_{\text {eff }}^{*}$ to $80 \%$ of the $K$ value.

\section{Automatic TARGeting By Visual SERVOING}

In this section we present the visual control law that we propose for automatically guiding the needle in order to reach a target point $O$ defined by the user at location ${ }^{w} \mathbf{T}_{o}=\left(t_{o x}, t_{o y}, t_{o z}\right)$ in the world frame.

As the target coordinates are expressed in the world frame $\mathcal{F}_{w}$ and not directly in the needle frame $\mathcal{F}_{n}$, it is more suitable to express the needle lateral angular control velocities in the fixed world frame $\mathcal{F}_{w}$. The lateral angular velocities applied to the needle frame will then be obtained from the lateral control velocities $\left({ }^{(n)} \omega_{x}^{*},{ }^{(n)} \omega_{y}^{*}\right)$ expressed in $\mathcal{F}_{w}$ thanks to the following relation:

$$
\left(\begin{array}{c}
{ }^{(n)} \omega_{x}^{*} \\
{ }^{(n)} \omega_{y}^{*}
\end{array}\right)={ }^{w} \mathbf{R}_{n_{[2 \times 2]}}{ }^{T}\left(\begin{array}{c}
{ }^{(w)} \omega_{x}^{*} \\
{ }^{(w)} \omega_{y}^{*}
\end{array}\right)
$$

where ${ }^{w} \mathbf{R}_{n_{[2 \times 2]}}{ }^{T}$ is the transpose of the [2×2] upper-left part of ${ }^{w} \mathbf{R}_{n}$. It follows that in order to control the needle in the world frame reference, we need to know the rotation matrix that describes the orientation of the needle frame with respect to the world frame. We will detail now the method we propose to estimate it and also how to reconstruct the full needle $3 \mathrm{D}$ pose from measurements.

\section{A. Needle $3 D$ pose estimation}

We consider that a current $3 \mathrm{D}$ volume observing the needle is available thanks to the use of a $3 \mathrm{D}$ ultrasound probe or a TDM or MRI imaging device. It is therefore possible to extract the voxels belonging to the shape of the needle by performing some image processing on the volume. We will not detail the method to extract the needle but the reader can refer to our previous work [17] where we presented a RANSAC-based algorithm to extract in real-time a flexible needle from a sequence of 3D ultrasound volumes. This extraction gives us the current location of the needle tip point denoted $N$ of coordinates ${ }^{w} \mathbf{T}_{n}=\left(t_{n x}, t_{n y}, t_{n z}\right)$ and its unitary orientation vector ${ }^{w} \mathbf{u}=\left(u_{x}, u_{y}, u_{z}\right)$ at its extremity both expressed in the volume cartesian frame that corresponds to the world frame $\mathcal{F}_{w}$. From these visual measures we can therefore only partially determine the current $3 \mathrm{D}$ pose of the needle frame with respect to the world frame that is described by the homogeneous matrix ${ }^{w} \mathbf{M}_{n}$ (see (1)). In fact, the missing information corresponds to the orientation of the needle around its z-axis since it is not possible to identify it from vision due to the tubular needle shape. However, this angle information is directly accessible from the duty-cycling low-level controller and corresponds to the absolute angle $\theta_{a}$ that is continuously measured by the actuator encoder. By describing the orientation of the needle ${ }^{w} \mathbf{R}_{n}$ with the Euler angles using the $\mathrm{x}-\mathrm{y}-\mathrm{z}$ convention where $(\phi, \psi, \gamma)$ are respectively the rotation angles around the $x, y$ and $z$ axis we obtained the following expression:

${ }^{w} \mathbf{R}_{n}=\left[\begin{array}{ccc}c_{\psi} c_{\gamma} & -c_{\psi} s_{\gamma} & s_{\psi} \\ s_{\phi} s_{\psi} c_{\gamma}+c_{\phi} s_{\gamma} & -s_{\phi} s_{\psi} s_{\gamma}+c_{\phi} c_{\gamma} & -s_{\phi} c_{\psi} \\ -c_{\phi} s_{\psi} c_{\gamma}+s_{\phi} s_{\gamma} & c_{\phi} s_{\psi} s_{\gamma}+s_{\phi} c_{\gamma} & -c_{\phi} c_{\psi}\end{array}\right]$

with the notation $c_{x}=\cos (x)$ and $s_{x}=\sin (x)$. As the third column corresponds directly to the needle unitary orientation vector ${ }^{w} \mathbf{u}$ extracted from the volume we can easily identify the $\phi$ and $\psi$ angles by the relations:

$$
\begin{aligned}
\phi & =\operatorname{atan}\left(-u_{y} / u_{z}\right) \\
\psi & =\operatorname{atan}\left(u_{x} /\left(c_{\phi} u_{z}-s_{\phi} u_{y}\right)\right)
\end{aligned}
$$

If the needle frame axes are initially aligned with the world frame axes before starting the 3D duty-cycling control process, then the angle $\gamma$ will correspond directly to the absolute angle $\theta_{a}$ measured by the actuator encoder during the needle trajectory evolution. Therefore by substituting $\gamma=\theta_{a}$ and (12) in (11) we obtain the estimated rotation matrix ${ }^{w} \widehat{\mathbf{R}}_{n}$. Consequently we also obtain the full homogeneous matrix ${ }^{w} \widehat{\mathbf{M}}_{n}$ that describes the estimated current pose of the needle frame with respect to the world frame.

\section{B. Visual servoing}

To perform the task we propose to apply a constant needle insertion velocity ${ }^{(n)} v_{z}=v_{c}$ and online adapt the angular control velocities $\left({ }^{(w)} \omega_{x}^{*},{ }^{(w)} \omega_{y}^{*}\right)$ by visual servoing in such a way to orientate the needle $\mathrm{z}$-axis toward the target. The set of visual features that we chose is $\mathbf{s}=\left(l_{x z}, l_{y z}\right)$ where $l_{x z}$ and $l_{y z}$ are respectively the distances between the target point $O$ and their orthogonal projections onto the needle line direction projected in the $\mathrm{x}-\mathrm{z}$ plane and $\mathrm{y}-\mathrm{z}$ plane of $\mathcal{F}_{w}$ as shown in Figure 2a. Let us denote $d_{x z}$ and $d_{y z}$ the distances of the projections of vector $\overrightarrow{N O}$ into the $\mathrm{x}$ $\mathrm{z}$ plane and $\mathrm{y}-\mathrm{z}$ plane of $\mathcal{F}_{w}$. We also define $\theta_{x}$ and $\theta_{y}$ as being respectively the angles around the world frame 
$\mathrm{x}$ and $\mathrm{y}$ axes that describe the relative orientation of the vector $\overrightarrow{N O}$ with respect to needle unitary orientation vector ${ }^{w} \mathbf{u}$ (see Figure 2a). Note that all these geometrical features can be easily computed from the measured 3D positions of the needle and target. The current visual features are then obtained by: $l_{x z}=d_{x z} \sin \left(\theta_{y}\right)$ and $l_{y z}=-d_{y z} \sin \left(\theta_{x}\right)$. The visual servoing task consists then in aligning the needle line direction on the target by regulating $\mathbf{s}$ to the desired value $\mathbf{s}^{*}=(0,0)$. As we previously mentioned, one important advantage of our new 3D duty-cycling strategy is that we can apply the classical framework of visual servoing [13]. Therefore we first determine the image jacobian $\mathbf{J}$ that relates the variation $\dot{\mathbf{s}}$ of the selected visual features to the needle velocity inputs and obtain the following expression from basic kinematic relationships:

$$
\begin{gathered}
\dot{\mathbf{s}}=\left(\begin{array}{c}
\dot{l_{x z}} \\
\dot{l_{y z}}
\end{array}\right)=\underbrace{\left[\begin{array}{ll}
\mathbf{J}_{\omega_{x y}} & \mathbf{J}_{v_{z}}
\end{array}\right]}_{=\mathbf{J}}\left(\begin{array}{c}
(w) \omega_{x} \\
(w) \omega_{y} \\
(n) v_{z}
\end{array}\right) \\
\text { with } \mathbf{J}_{\omega_{x y}}=\left[\begin{array}{cc}
0 & d_{x z} \cos \left(\theta_{y}\right) \\
-d_{y z} \cos \left(\theta_{x}\right) & 0
\end{array}\right], \mathbf{J}_{v_{z}}=\left[\begin{array}{c}
0 \\
0
\end{array}\right]
\end{gathered}
$$

We can note that the variation of the features are totally decoupled from the needle translational velocity applied in the needle frame and that they are independently coupled to the needle lateral angular velocities expressed in the world frame. This shows that the selected features are strongly suited for the required target alignment task since the constant translation velocity will note disturb the needle alignment task. Finally we applied the following classical control law that gives the desired control angular velocity inputs in such a way to obtain an exponential decrease of the visual error $\mathbf{e}=\mathbf{s}-\mathbf{s}^{*}$ :

$$
\left(\begin{array}{c}
(w) \omega_{x}^{*} \\
(w) \omega_{y}^{*}
\end{array}\right)=-\lambda \mathbf{J}_{\omega_{x y}}^{-1} \mathbf{e}
$$

where $\lambda>0$ is a proportional gain. The angular velocities are then expressed in the needle frame $\mathcal{F}_{n}$ thanks to the relation (10). The desired needle spin angle $\theta^{*}$ to accumulate and the desired effective curvature $K_{\text {eff }}^{*}$ are then computed using (8)-(9) and applied with the constant insertion velocity to our 3D duty-cycling low-level controller presented in Section IVB.

\section{EXPERIMENTAL RESULTS}

In this section we present experimental results of the automatic visual targeting task using our 3D duty-cycling new technique. We consider the images provided by two cameras in order to demonstrate the feasibility of our approach but other kinds of image modality as for example intraoperative 3D ultrasound images could be employed. The experiment setup is presented in Figure 2b. It is composed of a 6-DOF anthropomorphic robot holding a 22 gauge bevel-tip flexible needle whose length is $20 \mathrm{~cm}$. A home-made translucent gelatin phantom contained in a translucent box is employed to simulate a piece of organic soft tissues and is observed by two orthogonal video cameras. These cameras are connected

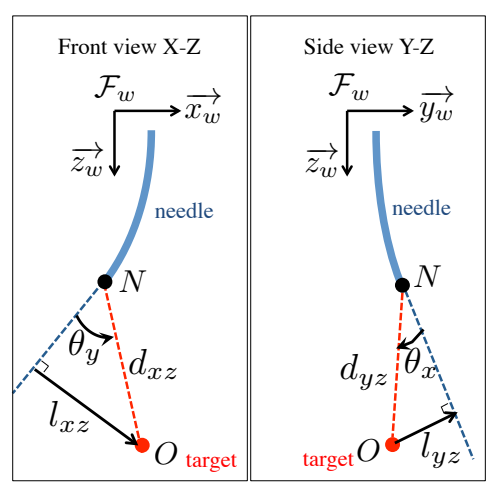

(a)

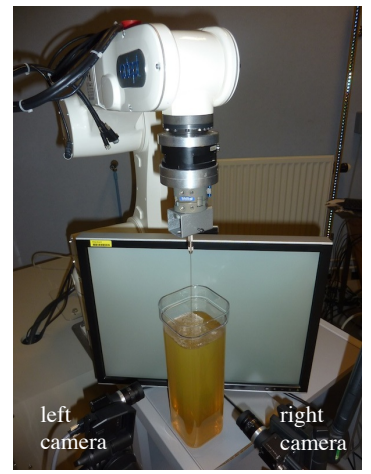

(b)
Fig. 2. (a) Geometry of selected visual features. (b) Experimental setup.

on the firewire interface of a PC equiped with a Dual-core 2.4 Ghz Intel Pentium that grabs the images at a frame rate of 15 images/second. A flat screen monitor is positioned behind the phantom in order to provide a scattered light inside it. Another firewire connexion of the PC is used to send the joint velocity control inputs to the robot and receive the joint measures. A software that we designed in $\mathrm{C}++$ (based on ViSP open source library [19]) is running on the PC to perform the needle motion control. This software realizes in a a fist thread the image processing and computes the visual servoing control law output (15). This later is continuously sent to a second thread that performs the $3 \mathrm{D}$ duty-cycling low-level process.

The intrinsic parameters of the two cameras were calibrated in order to allow the conversion from pixels to meters for a given distance to the phantom. In order to observe the whole trajectory of the needle, these distances were respectively fixed to $14 \mathrm{~cm}$ and $19 \mathrm{~cm}$ for the left and right cameras since their objective focal length were not identical. To perform a 3D localization of the needle during its insertion, we extract the needle tip meter coordinates from the two orthogonal images thanks to an image processing algorithm that automatically detects the shape of the needle. This algorithm consists in thresholding the images and applying few morphological operators (erosion and dilatation). For a lack of place we do not detail it more in depth since it is out of the topic of this paper. For all experiments the needle frame $\mathcal{F}_{n}$ was initially aligned with the frame $\mathcal{F}_{w}$ before the insertion.

In a first experiment, we do not apply our visual servoing scheme that relies on our 3D duty-cycling technique but we apply only a constant insertion velocity ${ }^{(n)} v_{z}^{*}=1 \mathrm{~mm} / \mathrm{s}$ to the needle. Figures $3 \mathrm{a}$ and $3 \mathrm{~b}$ show respectively the resulted trajectory observed in the left image (corresponding to the $\mathrm{y}-\mathrm{z}$ plane of $\mathcal{F}_{w}$ ) and the right image (corresponding to the $\mathrm{X}-\mathrm{Z}$ plane of $\mathcal{F}_{w}$ ). One can observe that the needle follows as expected its natural curvature. Note that the needle current location is surrounded in red by the image processing algorithm and that the other visible lines correspond in fact to few holes performed by the needle in the phantom during previous insertions. Moreover, the final vertical locations in pixels are not the same in the two images since the 


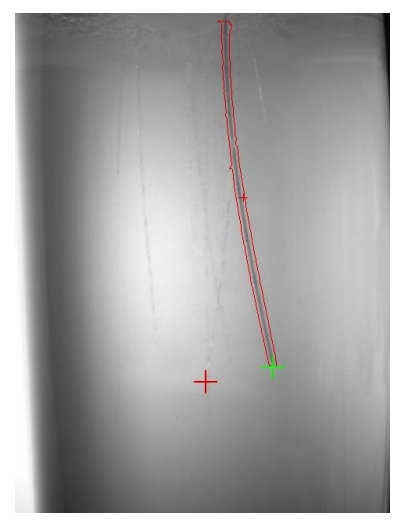

(a)

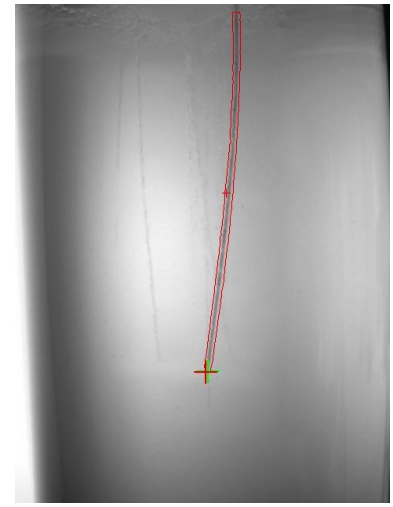

(c)

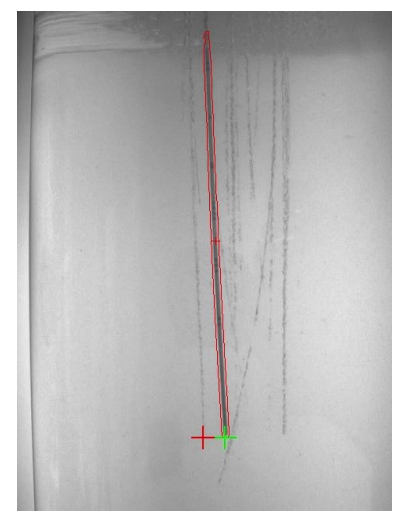

(b)

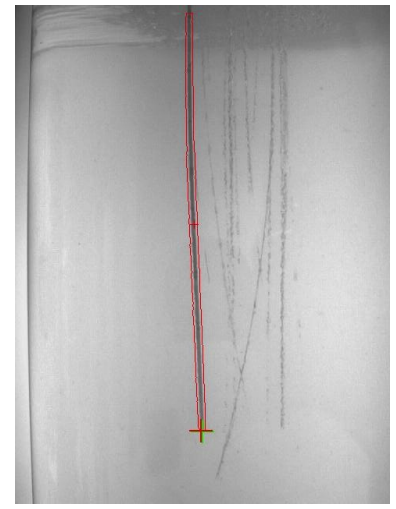

(d)
Fig. 3. (a) left camera (y-z plane) and (b) right camera (x-z plane) views of the needle trajectory obtained with a pure needle insertion motion. (c) left camera (y-z plane) and (d) right camera ( $x-z$ plane) views of the needle trajectory obtained with the targeting task by visual servoing. The target is represented by the red cross and needle tip by the green one.

distances from the cameras to the phantom are different but the final vertical coordinates in meter that are obtained after pixel-meter conversion (thanks to the calibrated intrinsic parameters of the cameras) are identical. From this first experiment, we coarsely estimate from the location of three $3 \mathrm{D}$ points on the needle arc trajectory the natural curvature of the needle and obtain $K=2$ meter $^{-1}$ (corresponding to a radius of 0.5 meter).

In a second experiment, we test our targeting task by applying the visual control law (15) and using our 3D dutycycling low-level controller with a constant insertion velocity of $1 \mathrm{~mm} / \mathrm{s}$. The nominal needle spin velocity that we use to compute the profile of the spin velocity ${ }^{(n)} \omega_{z}^{*}$ that is applied by our 3D duty-cycling low-level controller (see Section IV-B) was fixed to $\omega_{c}=2 \pi \mathrm{rad} / \mathrm{second}$. For this experiment the control gain was set empirically to the low value of $\lambda=0.02$ in order to obtain a smooth trajectory. Since we did not put a physical target in the phantom, we first inserted the needle manually in order to reach a position that we defined as being the target to reach. This allows us to set the target coordinates by clicking on the needle tip in the two images. Then we remove the needle and slightly displace the phantom in order to insert the needle in a new entry point of the phantom. Finally we apply the automatic targeting by visual servoing. Figures $3 c$ and $3 d$ present the

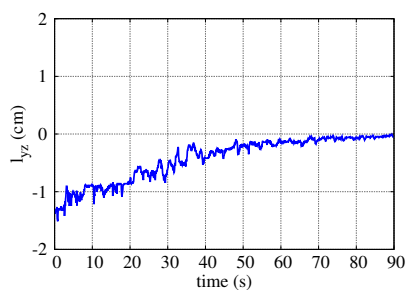

(a) Visual error $l_{y z}(\mathrm{~cm})$

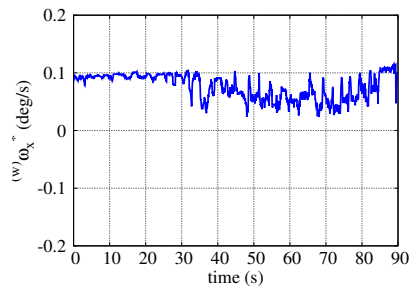

(c) Velocity $(w) \omega_{x}^{*}(\mathrm{deg} / \mathrm{s})$

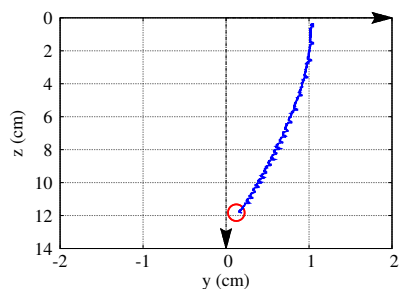

(e) $\mathrm{y}-\mathrm{z}$ needle trajectory $(\mathrm{cm})$

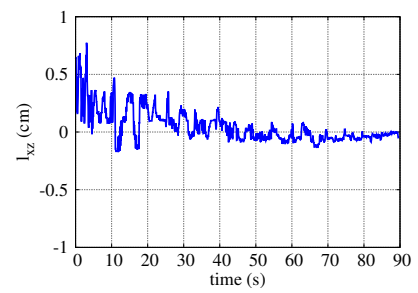

(b) Visual error $l_{x z}(\mathrm{~cm})$

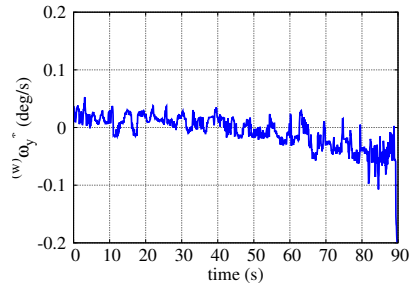

(d) Velocity $(w) \omega_{y}^{*}(\mathrm{deg} / \mathrm{s})$

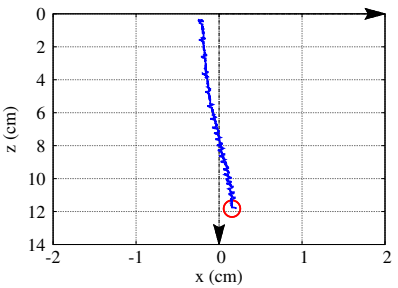

(f) $\mathrm{x}-\mathrm{z}$ needle trajectory $(\mathrm{cm})$

Fig. 4. Results of the targeting task by visual servoing: (a)-(b) visual features errors, (c)-(d) control angular velocities expressed in the world frame, (e)-(f) obtained needle trajectory in the $y-x$ and $x-z$ world frame planes. The red circle represents the target to reach.

obtained trajectory that is observed in the left and right images. One can see that the target depicted by the red cross is perfectly reached by the needle tip in the two images. The evolutions of the visual errors are presented in Figures $4 \mathrm{a}-4 \mathrm{~b}$ and correspond as expected to exponential decreases toward zero. Note that the noise is essentially due to the image processing algorithm and could be reduced with the use of a more efficient algorithm to extract the needle from the images. At the convergence the final positioning errors are $0.4 \mathrm{~mm}$ for $l_{x z}$ and $0.5 \mathrm{~mm}$ for $l_{y z}$. The lateral angular control velocities ${ }^{(w)} \omega_{x}^{*}$ and ${ }^{(w)} \omega_{y}^{*}$ of the needle expressed in the world frame are reported in Figures 4c-4d. One can see that the velocities decrease during the first 70 seconds and then increase when the needle reaches the target. This is due to the fact that the control law actively reacts to compensate the final increase of angles $\theta_{x}$ and $\theta_{y}$ when the needle tip get more and more close to the target in order to refine the alignment, but this is not an issue since the needle insertion motion is automatically stopped when the $\mathrm{z}$ component of the euclidean distance between the tip and target is null. Figures $4 \mathrm{e}-4 \mathrm{f}$ show the projection of the needle trajectory onto the y-z and x-z planes of $\mathcal{F}_{w}$ and the 3D trajectory is depicted in Figure 5a. Again, we can see that the target depicted by the red circles is accurately reached as it is also demonstrated in the Figure $5 \mathrm{~b}$ that presents the evolution of the needle-target distance. The evolution of the duty-cycling control ratio 


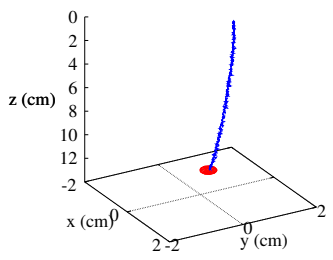

(a) Needle 3D trajectory $(\mathrm{cm})$

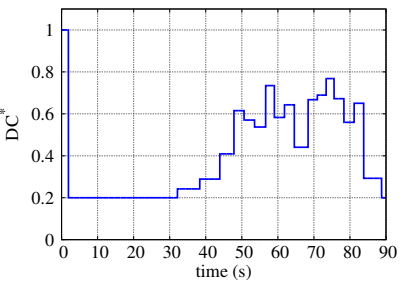

(c) Duty-cycling ratio $D C^{*}$

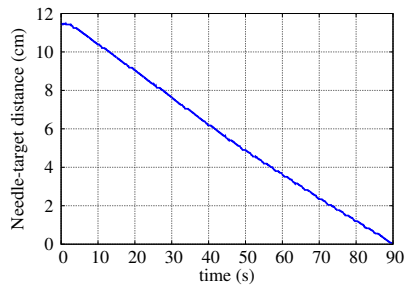

(b) Needle-target distance $(\mathrm{cm})$

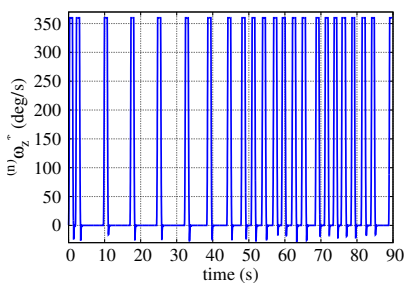

(d) Duty-cycling velocity $\omega_{z}^{*}(\mathrm{deg} / \mathrm{s})$
Fig. 5. Results of the targeting task by visual servoing: (a) obtained needle 3D trajectory, (b) evolution of the distance between the needle and target, (c) duty-cycling ratio and (d) spin angular velocity ${ }^{(n)} \omega_{z}^{*}$ applied by the low-level 3D duty-cycling controller.

$D C^{*}$ applied by our 3D duty-cycling low-level controller is reported in Figure 5c. We can observe that at the start of the targeting task $D C^{*}$ is low in order to apply an important curvature to the needle, then in the period from 40 to 70 seconds, it progressively increases to realize a more straight trajectory of the needle since this one becomes well aligned on the target. Then the ratio $D C^{*}$ drastically decreases when the needle reaches the target due to the final increase of $\theta_{x}$ and $\theta_{y}$ that we explained before. Figure $5 \mathrm{~d}$ shows the evolution of the desired spin angular velocity profile ${ }^{(n)} \omega_{z}^{*}$ applied by the duty-cycling low-level controller. We can see that the duration $T_{\text {trans }}$ of the pure insertion motion (phases when ${ }^{(n)} \omega_{z}^{*}=0$ ) progressively decreases while the needle direction becomes more and more aligned on the target. These results also experimentally demonstrate that the targeting task by visual servoing is robust to modelling errors since we do not consider the torsional effect of the needle in its kinematic model and we employ a coarse estimated value of its natural curvature that is not on-line adapted during the control.

\section{CONCLUSION}

In this paper we presented a new duty-cycling strategy that allows to control the insertion velocity and the two lateral angular velocities of the needle tip. This control is performed continuously at the rate of the duty-cycling period and allows therefore to steer the needle in 3D in opposite to the classical duty-cycling approach that is limited to planar bending. Experimental results demonstrate the efficiency of our approach to steer the needle to reach a 3D target without relying like other methods on a path planning that generates a 3D trajectory composed from successive oriented planar arcs. In fact, as our strategy allows to implement the classical task function approach, it will be possible in the future to design a multi-task control law to perform several hierarchical tasks at the same time like for example reaching a soft-tissue target while insuring obstacle avoidance and needle mechanical constraints. We are currently implementing the approach presented in this paper to automatically steer a flexible needle by ultrasound visual servoing thanks to the use of a $3 \mathrm{D}$ ultrasound probe.

\section{REFERENCES}

[1] K. Reed, A. Majewicz, V. Kallem, R. Alterovitz, K. Goldberg, N. Cowan, and A. Okamura, "Robot-assisted needle steering," IEEE Robotics and Automation Magazine, vol. 18, no. 4, pp. 35-46, December 2011.

[2] R. Webster III, J. Kim, N. Cowan, G. Chirikjian, and A. M. Okamura, "Nonholonomic modeling of needle steering," The International Journal of Robotics research, vol. 25, no. 5-6, pp. 509-525, 2006.

[3] S. Misra, K. Reed, B. Schafer, K. Ramesh, and A. Okamura, "Mechanics of flexible needles robotically steered through soft tissue," The International Journal of Robotics Research, vol. 29, no. 13, pp. 1640-1660, November 2010.

[4] D. Glozman and M. Shoham, "Image-guided robotic flexible needle steering," IEEE Trans. on Robotics, vol. 23, no. 3, pp. 459-467, 2007.

[5] V. Duindam, J. Xu, R. Alterovitz, S. Sastry, and K. Goldberg, "Threedimensional motion planning algorithms for steerable needles using inverse kinematics," The International Journal of Robotics Research, vol. 29, no. 7, pp. 789-800, 2010.

[6] R. Alterovitz, K. Goldberg, and A. Okamura, "Planning for steerable bevel-tip needle insertion through $2 \mathrm{~d}$ soft tissue with obstacles," in IEEE Int. Conf on Robotics and Automation, 2005, pp. 1640-1645.

[7] S. LaValle and J. Kuffner, "Randomized kinodynamic planning," in IEEE Int. Conf. on Robotics and Automation, vol. 1, 1999, pp. 473479.

[8] R. Alterovitz, T. Simon, and K. Goldberg, "The stochastic motion roadmap: A sampling framework for planning with markov motion uncertainty," In Robotics: Science and Systems III, W. Burgard and al. (Eds.), MIT Press, pp. 233-241, 2008.

[9] N. Wood, K. Shahrour, M. Ost, and C. Riviere, "Needle steering system using duty-cycled rotation for percutaneous kidney access," in IEEE Int. Conf on Engineering in Medicine and Biology Society (EMBS), 2010, pp. 5432-5435.

[10] D. Minhas, J. Engh, M. Fenske, and C. Riviere, "Modeling of needle steering via duty-cycled spinning," in IEEE Int. Conf. on Engineering in Medicine and Biology Society, 2007, pp. 2756-2759.

[11] J. Xu, V. Duindam, R. Alterovitz, and K. Goldberg, "Motion planning for steerable needles in $3 \mathrm{~d}$ environments with obstacles using rapidlyexploring random trees and backchaining," in IEEE Int. Conf on Automation Science and Engineering, 2008, pp. 41-46.

[12] M. Bernardes, B. Adorno, P. Poignet, and G. Borges, "Robot-assisted automatic insertion of steerable needles with closed-loop imaging feedback and intraoperative trajectory replanning," Mechatronics, vol. 23 , pp. 630-645, 2013.

[13] F. Chaumette and S. Hutchinson, "Visual servo control, part i: Basic approaches," IEEE Robotics and Automation Magazine, vol. 13, no. 4, pp. 82-90, December 2006.

[14] C. Samson, B. Espiau, and M. Le Borgne, Robot control: The task function approach. Oxford University Press, 1991.

[15] C. Nadeau, A. Krupa, N. Zemiti, P. Poignet, and J. Gangloff, "Active stabilization of ultrasound image for robotically-assisted medical procedures," in Hamlyn Symposium on Medical robotics, London, UK, July 2012.

[16] C. Nadeau and A. Krupa, "Intensity-based ultrasound visual servoing: modeling and validation with $2 \mathrm{~d}$ and $3 \mathrm{~d}$ probes," IEEE. Trans. on Robotics, vol. 29, no. 4, pp. 1003-1015, August 2013.

[17] P. Chatelain, A. Krupa, and M. Marchal, "Real-time needle detection and tracking using a visually servoed $3 \mathrm{~d}$ ultrasound probe," in IEEE Int. Conf. on Robotics and Automation, Karlsruhe, Germany, May 2013, pp. 1668-1673.

[18] M. Bernardes, B. Adorno, G. A. Borges, and P. Poignet, "3d robust online motion planning for steerable needles in dynamic workspaces using duty-cycled rotation," Journal of Control, Automation and Electrical Systems, 2014.

[19] E. Marchand, F. Spindler, and F. Chaumette, "Visp for visual servoing: a generic software platform with a wide class of robot control skills," IEEE Robotics and Automation Magazine, vol. 12, no. 4, pp. 40-52, December 2005. 